Abstract \#1787, 236th ECS Meeting

\title{
Electrical Properties of Monocrystalline Thin Film Si for Solar Cells Fabricated By Rapid Vapor Deposition with Nano-Surface Controlling Double Layer Porous $\mathrm{Si}$ in $\mathbf{H}_{2}$
}

\author{
Ryotaro Shibahara $^{\mathrm{a}}$, Kei Hasegawa ${ }^{\mathrm{a}}$, Alain Fave ${ }^{\mathrm{b}}$, Erwann Fourmond ${ }^{\mathrm{b}}$, Suguru Noda ${ }^{\mathrm{c}}$, Manabu Ihara ${ }^{\mathrm{a}}$

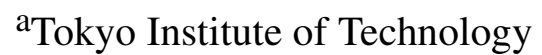 \\ ${ }^{\mathrm{b}}$ Institut des Nanotechnologies de Lyon (INSA-Lyon) \\ ${ }^{\mathrm{c}}$ Waseda University
}

\section{Introduction}

To reduce the Si thickness with maintaining the high quality is a promising approach to reduce the cost of monocrystalline Si solar cell. A major method to fabricate monocrystalline thin Si is epitaxy by Chemical Vapor Deposition (CVD) and Layer Transfer Process (LTP) as shown in Fig. 1. A seed layer and a sacrificial layer such as double layer porous Si (DLPS) which consist of a Low Porous Layer (LPL) and a High Porous Layer (HPL) are fabricated on the surface of a monocrystalline Si wafer, and then Si is epitaxially deposited on the seed layer. This wafer can then be reused in LTP, thus further reducing the material cost of these Si cells. There remain two challenging issues: (i) crystal defect introduced during epitaxy caused by the roughness of the seed layer ${ }^{1)}$ and (ii) low deposition rate and yield of epitaxy by CVD. To solve problem (i), we proposed a Zone Heating Recrystallization (ZHR) method ${ }^{2}$ ) to smoothen the DLPS surface as shown in Fig.2. The structure of the DLPS surface can be modified by using an upper lamp heater to scan the surface in one direction and a bottom heater to pre-heat Si substrate. To solve problem (ii), we proposed a Rapid Vapor Deposition (RVD) method ${ }^{3)}$ as shown in Fig.3. By depositing Si under a high vapor pressure by heating the source Si to over $2000^{\circ} \mathrm{C}$, the deposition rate of over $10 \mu \mathrm{m} / \mathrm{min}$ with a higher yield is achieved.

By applying both the ZHR and RVD methods, we successfully reduced the roughness of a DLPS surface and obtained monocrystalline Si with Si wafer level. The critical effect of lowering the roughness of a DLPS surface to $\mathrm{R}_{\mathrm{ms}}<0.3 \mathrm{~nm}$ was

confirmed ${ }^{144)}$. In this work, to fabricate high quality solar cells, we investigated the key factors in improving the roughness of a DLPS surface and evaluated the semiconductor properties of monocrystalline thin film Si.

\section{Experiment}

DLPS was prepared by 2-step anodization of p-type monocrystalline $\mathrm{Si}(100)$ wafers $(\leqq 0.005 \Omega \cdot \mathrm{cm})$ with $2 \mathrm{~mA} / \mathrm{cm}^{2}$ and $415 \mathrm{~s}$ for LPL and $200 \mathrm{~mA} / \mathrm{cm}^{2}$ and $5 \mathrm{~s}$ for HPL, respectively. The prepared DLPS were thermally treated by ZHR with adjusting the heat levels of both the upper and bottom heater and the scan rate of the upper heater under $\mathrm{H}_{2}(0 \sim 5 \%) / \mathrm{N}_{2}$ atmosphere to investigate the effect of surface oxidation.

RVD were carried out with using p-type monocrystalline $\mathrm{Si}(100)(1-10 \Omega \cdot \mathrm{cm})$ wafers as the source. The thickness of Si was required to be $>40 \mu \mathrm{m}$ to obtain the sufficient light absorbance, but in our RVD apparatus, only $<10 \mu \mathrm{m}$-thick Si can be fabricated in one deposition due to the structure of the source boats, so Si was epitaxially deposited on DLPS by RVD in 5 time depositions and the film ( $48 \mu \mathrm{m}$-thick) (Sample-1) was obtained. To increase the deposition rate, the tungsten boat to put $\mathrm{Si}$ was modified and the film with $35 \mu \mathrm{m}$-thick was obtained in one time deposition (Sample-2).

The roughness of the DLPS surface were analyzed using atomic force microscopy. To measure the mobility and the carrier

concentration, Si was epitaxially deposited directly on non-doped $\mathrm{Si}(100)(\geqq 1000 \Omega \cdot \mathrm{cm})$ wafer and the hall effect measurement was carried out. The lifetime of obtained $\mathrm{Si}$ films were measured after passivated with $\mathrm{Al}_{2} \mathrm{O}_{3}$ film on both sides by atomic layer deposition.

\section{Results and discussion}

The minimum roughness of DLPS surface and the heating amount which minimize the roughness of DLPS surface was decreased by changing the atmosphere from $\mathrm{N}_{2}$ to $\mathrm{H}_{2} / \mathrm{N}_{2}$. It is supposed that the structural change occurs easier by inhibiting the effect of oxidation, possibly due to the presence of $\mathrm{H}_{2}$.

The life time of Sample-2 was much higher than that of Sample-1. In the multiple RVD process, a native oxide film seemed to be formed on the surface of a Si film during re-setting sources in air atmosphere. Thus, a thin Si with almost wafer-level quality was obtained by increase the thickness by RVD in one deposition.

\section{Conclusion}

The importance to inhibit the effect of oxidation during ZHR was clarified. Nearly wafer-level, $35 \mu \mathrm{m}$-thick Si was obtained by RVD in one time deposition by increasing deposition rate.

\section{Acknowledgements}

The part of this research was supported by KAKENHI (KIBAN A) and Center for Low Carbon Society Strategy (LCS), JST.

1)K.Hasegawa,C.Takazawa,M.Fujita,S.Noda and M.Ihara,CrystEngComm,20(13),1774-1778(2018).

2)A.Lukianov,K.Murakami,C.Takazawa and M.Ihara,Appl.Phys.Lett.,108(213904),1-4(2016).

3)Y.Yamasaki,K.Hasegawa,T.Osawa and S.Noda,CrystEngComm,18,3404-3410(2016).

4)C.Takazawa,M.Fujita,K.Hasegawa,A.Lukianov,X.Zhang,S.Noda and M.Ihara,ECS Transaction,75(31),11-23(2016). 


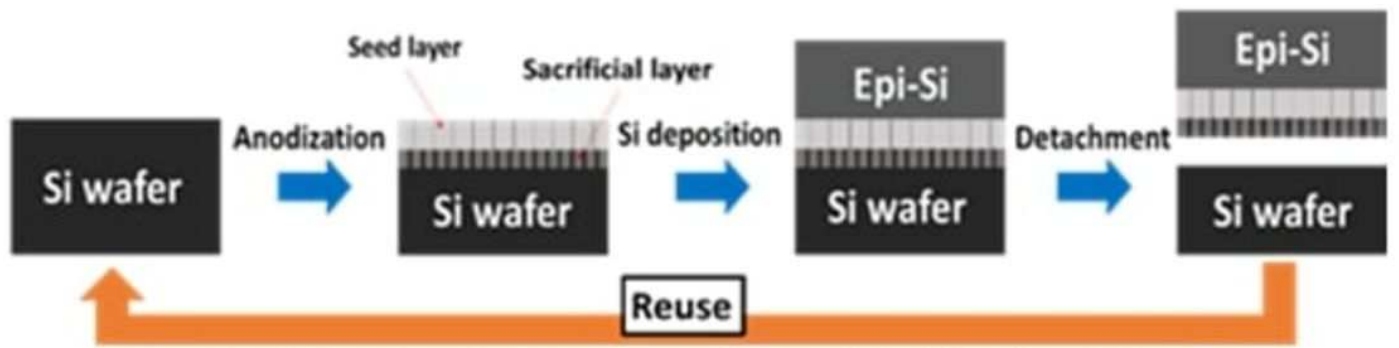

Fig.1 Fabrication process for a thin film by LTP

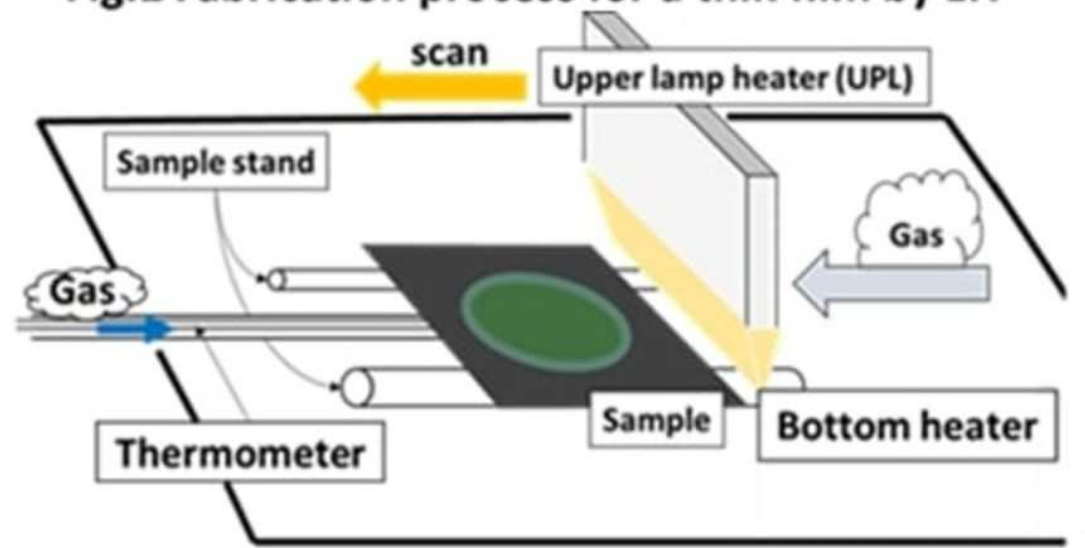

Fig.2 Schematic view of ZHR method ${ }^{2)}$

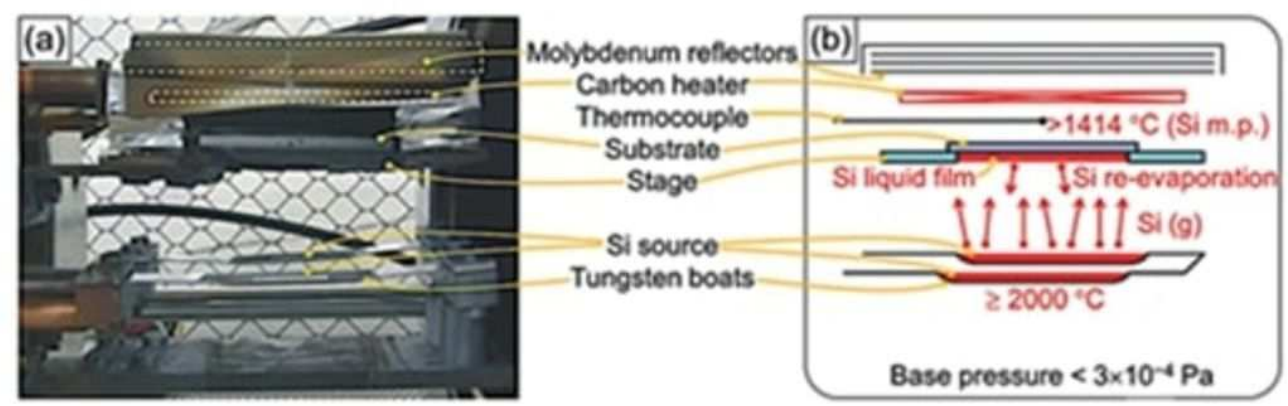

Fig.3 Schematic view of RVD method ${ }^{3)}$ 